\title{
CLINICAL NOTE ON FACIES QUADRIPLEGIAE
}

\author{
By Dr A. Ohry and Professor R. Rozin \\ Neurological Rehabilitation Department, Chaim Sheba Medical Centre, Tel Hashomer, \\ Israel
}

In our National Centre for the treatment of spinal cord injured patients, we have had the opportunity of observing hundreds of patients over the years. It is our impression that quadriplegia patients tend slowly to develop certain common facial characteristics. We suggest a new descriptive term which tries to summarise these characteristics: 'Facies Quadriplegiae'.

The common characteristics are the following: The patient's general appearance is usually dull and pale. During the immobilisation period his energy requirements and food intake are reduced: he usually loses weight, thus tending to appear thin, often with sunken cheeks. Pallor is due to slightly reduced haemoglobin concentration and less frequent exposure to the sun. We also observe in many of the patients sparse hair resulting from partial hair loss, a phenomenon also seen in astronauts who have spent time in a weightless environment. Because of severe paralysis, the only muscles remaining intact in high cervical cord injured persons are those of the face and neck. Occasionally, excessive movement of the whole head, of shoulder elevation or of the facial movements are seen, while the remaining intact muscles become hypertrophied. Accessory respiratory muscles such as the scaleni, the sternocleidomastoid and the alae nasi act vigorously. Some of these patients also have Horner's syndrome (ptosis, anhydrosis and mydriasis). Paralytic vasodilo also causes conjunctival injection. Bilateral ptosis is occasionally seen. The skin is often oily and sometimes hyperhydrotic, and acne is common. Due to nasal congestion (Guttmann's sign), the voice is usually nasal and speech becomes halting because of shallow, rapid respiration. Autonomic dysreflexia may cause adrenergic crises; catecholamines are released in excess resulting in hypertension, bradycardia, sweating, trembling, pin-point pupils, redness of the face and goose flesh (cutis anserina). The 'Facies Quadriplegiae' often expresses helplessness, loneliness, depression etc.

We hope that this short communication will encourage others to identify additional features which characterise the quadriplegic facies. 